\title{
Numerical Simulation of the Fractional Dispersion Advection Equations Based on the Lattice Boltzmann Model
}

\author{
Boyu Wang $\mathbb{D}^{1},{ }^{1}$ Jianying Zhang, ${ }^{2}$ and Guangwu Yan ${ }^{1}$ \\ ${ }^{1}$ College of Mathematics, Jilin University, Changchun 130012, China \\ ${ }^{2}$ School of Mathematics and Statistics, Changchun University of Technology, Changchun 130012, China \\ Correspondence should be addressed to Boyu Wang; bywang_jlu@126.com
}

Received 25 May 2020; Revised 12 July 2020; Accepted 17 July 2020; Published 15 September 2020

Academic Editor: Jefferson L. M. A. Gomes

Copyright (c) 2020 Boyu Wang et al. This is an open access article distributed under the Creative Commons Attribution License, which permits unrestricted use, distribution, and reproduction in any medium, provided the original work is properly cited.

The fractional dispersion advection equations (FDAEs) have recently attracted considerable attention due to their extensive application in the fields of science and engineering. For example, it has been shown that the anomalous solute transport behaviour that exists in hydrology can be well explained by introducing FDAEs. Therefore, the study of FDAEs has profound significance for understanding real transport phenomena in nature. Nevertheless, the existing algorithms for the FDAEs are generally intricate and costly. Therefore, exploiting an efficient solution technique has been a concern for scientists. In an effort to overcome this challenge, a promising lattice Boltzmann (LB) model for the FDAEs is presented in this paper. The Riemann-Liouville definition and the Grünwald-Letnikov definition are introduced for the time derivatives. In addition, Chapman-Enskog analysis is applied to recover the FDAEs. To test the validity of the model, three numerical examples are carried out. In addition, a comparative study of the proposed model and the classical implicit finite difference scheme is also conducted. The numerical results show that the model is suitable for simulating FDAEs.

\section{Introduction}

To overcome certain disadvantages of lattice gas automata, such as the lack of Galilean invariance and the undesired statistical noise, the LB method was developed by introducing statistically averaged particle distribution functions instead of Boolean variables. The method's popularity in recent years can be attributed to its numerical stability, algorithmic parallelism, and programming simplicity. As a mesoscopic numerical method, it has shown its strength in simulating complex flow systems [1-3].

In addition, for nonlinear partial differential equations, the LB method has also become an effective numerical solver. LB models have widely been studied for various equations, including wave equations $[4,5], \mathrm{KdV}$ equations [6], Kuramoto-Sivashinsky equations [7], nonlinear anisotropic convection-diffusion equations [8], Gross-Pitaevskii equations $[9,10]$, nonlinear Schrödinger equations [11-13], and Ginzburg-Landau equations [14, 15].

Fractional partial differential equations (FPDEs) have gained extensive attention in the field of scientific research due to their applications in physical systems [16-18], chemical reactions [19], life sciences [20], computer sciences [21], digital cryptography systems [22], secure communications [23], and financial models [24]. Numerous studies have been conducted to construct several methods for solving FPDEs [25-33].

The authors of the current work are more concerned about the LB approaches for FPDEs. To date, a number of related works have been documented in the literature. For example, Xia et al. developed a multispeed LB model for the FPDE and successfully simulated the anomalous superdiffusion phenomenon [34]. An LB model was presented for subdiffusion equations in 2016 [35]. The numerical examples proposed by Zhang and Yan showed that the scheme had first-order accuracy. Zhou et al. simulated fractional advection-diffusion equations based on the LB model [36]. The model with second-order accuracy successfully predicted the mass transport in hydrological systems. In 2018, Dai et al. constructed an LB model for space-fractional reaction-diffusion equations with nonlinear source terms [37]. Wang et al. proposed a two-time relaxation LB model to describe 
fractional advection-dispersion equations and reproduced the solute transport in soils and aquifers [38]. In 2019, a multiple-relaxation-time (MRT) LB method for the spacefractional advection-diffusion equation was carried out by Cartalade et al. [39]. They successfully overcame the instability caused by the anisotropic diffusion tensor. In the same year, Du et al. performed the LB model for time subdiffusion equations in the Caputo sense [40]. In 2020, Du and Liu established an LB model with double-distribution functions for solving the fractional advection-diffusion problem coupled with the incompressible Navier-Stokes equations [41].

In this paper, the following one-dimensional FDAE of the form given by equation (1) is considered:

$$
\begin{aligned}
\frac{\partial u(x, t)}{\partial t}= & \left(A \frac{\partial^{1-\gamma}}{\partial t^{1-\gamma}}+B \frac{\partial^{1-\gamma}}{\partial t^{1-\gamma}}\right) \frac{\partial^{2}}{\partial x^{2}} u(x, t) \\
& -F \frac{\partial^{1-\gamma}}{\partial t^{1-\gamma}} \frac{\partial}{\partial x} u(x, t)+q(x, t),
\end{aligned}
$$

where $\gamma, v \in(0,1) ; A, B$, and $F$ are the parameters that need to be determined based on specific problems, and $q(x, t)$ is the source term. From equation (1), we can clearly observe that both the dispersion term and the advection term involve time-fractional derivatives. This equation plays a significant role in anomalous diffusion problems $[17,42]$. However, the complexity of this FDAE makes most of the classical numerical algorithms inefficient and intricate. Exploiting an appropriate method for solving equation (1) is still an arduous task for scientists. The LB method has emerged as an active candidate in the past three decades and seems to be a promising tool to study this FDAE. However, there has been no relevant $\mathrm{LB}$ model that can handle this equation to date. Based on the above description, we intend to propose an effective LB algorithm to investigate the FDAE in this research.

To apply the LB model, the Riemann-Liouville definition is employed for the time-fractional derivative terms and is given by the following equation:

$$
\begin{aligned}
D_{t}^{1-\gamma} u(x, t) & =\frac{\partial^{1-\gamma}}{\partial t^{1-\gamma}} u(x, t) \\
& =\frac{1}{\Gamma(\gamma)} \frac{\partial}{\partial t} \int_{0}^{t} \frac{u(x, \tau)}{(t-\tau)^{1-\gamma}} \mathrm{d} \tau, \quad 0<\gamma<1 .
\end{aligned}
$$

Denote

$$
\begin{aligned}
\tilde{u}(x, t) & =D_{t}^{1-\gamma} u(x, t)=\frac{\partial^{1-\gamma}}{\partial t^{1-\gamma}} u(x, t) \\
& =\frac{1}{\Gamma(\gamma)} \frac{\partial}{\partial t} \int_{0}^{t} \frac{u(x, \tau)}{(t-\tau)^{1-\gamma}} \mathrm{d} \tau, \\
\bar{u}(x, t) & =D_{t}^{1-\gamma} u(x, t)=\frac{\partial^{1-\nu}}{\partial t^{1-\nu}} u(x, t) \\
& =\frac{1}{\Gamma(\nu)} \frac{\partial}{\partial t} \int_{0}^{t} \frac{u(x, \tau)}{(t-\tau)^{1-\nu}} \mathrm{d} \tau .
\end{aligned}
$$

Therefore, equation (1) can be rewritten as follows:

$$
\frac{\partial u(x, t)}{\partial t}+F \frac{\partial}{\partial x} \widetilde{u}(x, t)=\frac{\partial^{2}}{\partial x^{2}}[A \widetilde{u}(x, t)+B \bar{u}(x, t)]+q(x, t) .
$$

The integral form is sometimes inconvenient for solving numerically. Hence, we apply the Grünwald-Letnikov definition to convert equations (3a) and (3b) into a series form:

$$
\begin{aligned}
\widetilde{u}(x, t) & =D_{t}^{1-\gamma} u(x, t) \\
& =\lim _{\Delta t \longrightarrow 0} \frac{\Delta t^{-1+\gamma}}{\Gamma(-1+\gamma)} \sum_{i=0}^{[t / \Delta t]} \frac{\Gamma(i-1+\gamma)}{\Gamma(i+1)} u(x, t-i \Delta t) \\
\bar{u}(x, t) & =D_{t}^{1-v} u(x, t) \\
& =\lim _{\Delta t \rightarrow 0} \frac{\Delta t^{-1+v}}{\Gamma(-1+\nu)} \sum_{i=0}^{[t / \Delta t]} \frac{\Gamma(i-1+\nu)}{\Gamma(i+1)} u(x, t-i \Delta t)
\end{aligned}
$$

The remaining part of the paper is constructed as follows. In Section 2, an LB model for FDAEs is presented. In Section 3 , three numerical experiments are carried out to demonstrate the validity of the proposed model. In Section 4, based upon the results, conclusions are made and presented.

\section{Model Formulation}

For a one-dimensional LB equation with the BhatnagarGross-Krook collision term,

$$
\begin{aligned}
f_{\alpha}\left(x+e_{\alpha} \Delta t, t+\Delta t\right)-f_{\alpha}(x, t) \\
=-\frac{1}{\tau}\left[f_{\alpha}(x, t)-f_{\alpha}^{\mathrm{eq}}(x, t)\right]+\Omega_{\alpha}(x, t),
\end{aligned}
$$

where $f_{\alpha}(x, t)$ is the discrete distribution function, $e_{\alpha}$ is the discrete velocity in the $\alpha$ direction, $\Delta t$ represents the time step of the model, $\tau$ represents the relaxation time, and $f_{\alpha}^{e q}(x, t)$ is the local equilibrium distribution function, which should meet the conservation law given by

$$
\sum_{\alpha} f_{\alpha}^{\mathrm{eq}}(x, t)=\sum_{\alpha} f_{\alpha}(x, t) \text {. }
$$

$\Omega_{\alpha}(x, t)$ is called the additional term, which can be expanded as follows:

$$
\Omega_{\alpha}=\sum_{n=1}^{\infty} \varepsilon^{n} \Omega_{\alpha}^{(n)}
$$

where $\varepsilon$ is a dimensionless expansion parameter that numerically equals $\Delta t$. Therefore, equation (6) can be rewritten as

$$
\begin{aligned}
f_{\alpha}\left(x+e_{\alpha} \varepsilon, t+\varepsilon\right)-f_{\alpha}(x, t) \\
=-\frac{1}{\tau}\left[f_{\alpha}(x, t)-f_{\alpha}^{\mathrm{eq}}(x, t)\right]+\Omega_{\alpha}(x, t) .
\end{aligned}
$$


It is feasible to assume that the time step of our model $\Delta t=\varepsilon$ is a small parameter. Therefore, we can employ the Taylor series expansion and Chapman-Enskog expansion [43]:

$$
f_{\alpha}\left(x+e_{\alpha} \varepsilon, t+\varepsilon\right)-f_{\alpha}(x, t)=\sum_{n=1}^{\infty} \frac{\varepsilon^{n}}{n !}\left(\frac{\partial}{\partial t}+\frac{\partial}{\partial x} e_{\alpha}\right)^{n} f_{\alpha}(x, t),
$$

$$
f_{\alpha}(x, t)=\sum_{n=0}^{\infty} \varepsilon^{n} f_{\alpha}^{(n)},
$$

where $f_{\alpha}^{(0)}=f_{\alpha}^{\text {eq }}$. In addition, we introduce $t_{n}$ as time scales:

$$
\begin{aligned}
& t_{n}=\varepsilon^{n} t, \quad n=0,1,2, \ldots, \\
& \frac{\partial}{\partial t}=\sum_{n=0}^{\infty} \varepsilon^{n} \frac{\partial}{\partial t_{n}}
\end{aligned}
$$

Now, substituting equations (8), (10), (11), and (12b) into equation (9), separating and reorganizing the terms based on different orders of $\varepsilon$, we can derive the following equations:

$$
\begin{aligned}
& O(\varepsilon): \Delta f_{\alpha}^{(0)}=-\frac{1}{\tau} f_{\alpha}^{(1)}+\Omega_{\alpha}^{(1)}, \\
& O\left(\varepsilon^{2}\right): \frac{\partial}{\partial t_{1}} f_{\alpha}^{(0)}+C_{2} \Delta^{2} f_{\alpha}^{(0)}+\tau \Delta \Omega_{\alpha}^{(1)}=-\frac{1}{\tau} f_{\alpha}^{(2)}+\Omega_{\alpha}^{(2)},
\end{aligned}
$$

where the partial differential operator $\Delta=\left(\partial / \partial t_{0}\right)+$ $e_{\alpha}(\partial / \partial x) \cdot C_{2}$ is a polynomial of $\tau$ given as

$$
C_{2}=-\tau+\frac{1}{2} \text {. }
$$

For the purpose of simulating FDAEs, the macroscopic variable $u(x, t)$ should be defined as

$$
u(x, t)=\sum_{\alpha} f_{\alpha}(x, t) .
$$

According to the definition of macroscopic variable equation (15), the conservation law given by equation (7), and the Chapman-Enskog expansion given by equation (11), we can derive

$$
\begin{aligned}
\sum_{\alpha} f_{\alpha}^{(0)}(x, t) & =u(x, t), \\
\sum_{\alpha} f_{\alpha}^{(n)} & =0, \quad n \geq 1 .
\end{aligned}
$$

The first two moments of $f_{\alpha}^{(0)}(x, t)$ are defined and selected as

$$
\begin{aligned}
& m^{(0)}(x, t)=\sum_{\alpha} f_{\alpha}^{(0)}(x, t) e_{\alpha}=F \widetilde{u}, \\
& \pi^{(0)}(x, t)=\sum_{\alpha} f_{\alpha}^{(0)}(x, t) e_{\alpha}^{2}=\eta(A \widetilde{u}+B \bar{u}),
\end{aligned}
$$

where the parameter $\eta$ is given by

$$
\eta=-\frac{1}{\varepsilon C_{2}}
$$

Then, the FDAE with second-order accuracy of the truncation error can be recovered:

$$
\frac{\partial u}{\partial t}+F \frac{\partial \widetilde{u}}{\partial x}=\frac{\partial^{2}}{\partial x^{2}}(A \widetilde{u}+B \bar{u})+q(x, t)+O\left(\varepsilon^{2}\right) .
$$

The detailed recovery process can be found in Appendix. Combining equations (16a), (17a), and (17b), the determination of equilibrium distribution functions is straightforward. In this paper, the D1Q3 LB model is employed, in which the discrete velocities are selected as $e_{\alpha}=\{0, c,-c\}$, where $c$ represents the lattice speed. The solutions to equilibrium distribution functions are given by

$$
\begin{aligned}
& f_{0}^{(0)}=u-\frac{\eta}{c^{2}}(A \widetilde{u}+B \bar{u}), \\
& f_{1}^{(0)}=\frac{1}{2}\left[\frac{\eta}{c^{2}}(A \widetilde{u}+B \bar{u})+\frac{1}{c} F \widetilde{u}\right], \\
& f_{2}^{(0)}=\frac{1}{2}\left[\frac{\eta}{c^{2}}(A \widetilde{u}+B \bar{u})-\frac{1}{c} F \widetilde{u}\right] .
\end{aligned}
$$

\section{Numerical Examples}

Example 1. Consider the following one-dimensional FDAE:

$$
\frac{\partial u(x, t)}{\partial t}=A \frac{\partial^{1-\gamma}}{\partial t^{1-\gamma}} \frac{\partial^{2}}{\partial x^{2}} u(x, t)-A \frac{\partial^{1-\gamma}}{\partial t^{1-\gamma}} \frac{\partial}{\partial x} u(x, t)+q(x, t),
$$

where $0<\gamma<1$ and $A=0.001$. The variables $x, t \in[0,1]$. The source term $q(x, t)$ is denoted in the following equation:

$$
q(x, t)=e^{x} t^{\gamma}(1+\gamma) .
$$

The initial and boundary conditions are given by

$$
\begin{aligned}
& u(x, 0)=0, \\
& u(0, t)=t^{1+\gamma}, \\
& u(1, t)=e t^{1+\gamma} .
\end{aligned}
$$

The exact solution to the problem is given by

$$
u(x, t)=e^{x} t^{1+\gamma} .
$$

By employing equation (5a), the initial and boundary conditions of variable $\widetilde{u}$ are given by

$$
\begin{aligned}
& \widetilde{u}(x, 0)=0, \\
& \widetilde{u}(0, t)=\frac{\Delta t^{-1+\gamma}}{\Gamma(-1+\gamma)} \sum_{i=0}^{[t / \Delta t]} \frac{\Gamma(i-1+\gamma)}{\Gamma(i+1)}(t-i \Delta t)^{1+\gamma}, \\
& \widetilde{u}(1, t)=\frac{\Delta t^{-1+\gamma}}{\Gamma(-1+\gamma)} \sum_{i=0}^{[t / \Delta t]} \frac{\Gamma(i-1+\gamma)}{\Gamma(i+1)}(t-i \Delta t)^{1+\gamma} e .
\end{aligned}
$$


From equation (3a), the exact solution of variable $\tilde{u}$ is obtained:

$$
\widetilde{u}(x, t)=\frac{\Gamma(\gamma+2)}{\Gamma(2 \gamma+1)} e^{x} t^{2+\gamma} .
$$

In Figure 1(a), the exact solutions and LB numerical solutions for $t=1.0$ are shown. The parameters are as follows: $\gamma=0.5$, total lattice number $M=100, \Delta x=0.01$, $\Delta t=0.01, c=1$, and $\tau=0.908$. The solutions of variables $u(x, t)$ and $\tilde{u}(x, t)$ are both presented, which illustrate that the LB solutions agree with the exact solutions.

In Figures 1(b) and 1(c), surface plots of the absolute and relative errors are presented, respectively. The definitions of the absolute errors and relative errors are given by equations (27a) and (27b), respectively:

$$
\begin{aligned}
& E_{a}=\left|u^{N}-u^{E}\right|, \\
& E_{r}=\frac{\left|u^{N}-u^{E}\right|}{\left|u^{E}\right|} .
\end{aligned}
$$

In equations (27a) and (27b), $u^{N}$ represents the solutions calculated using the LB model presented in this paper and $u^{E}$ represents the exact solutions.

To show the errors more clearly, we also plot the absolute and relative errors when $t=1.0$ in Figure 1(d). From Figures $1(\mathrm{~b})-1(\mathrm{~d})$, we can observe that the absolute errors are limited within the value of $2.0 \times 10^{-2}$, while the relative errors are within the value of $8.0 \times 10^{-2}$ from $t=0.01$ to $t=1.0$. When $t=1.0$, the relative errors are approximately $7.8 \times 10^{-3}$. These results are acceptable.

To analyse the relationship between the errors and lattice size $\Delta x$, a log-log graph is plotted for $t=1.0$ (see Figure $1(\mathrm{e})$ ). The regression fit is also employed to quantify the trend of errors. The results show that $\log _{10} E_{a}=1.008 \log _{10} \Delta x+0.339$ and $\log _{10} E_{r}=1.013 \log _{10} \Delta x-0.083$, which indicate that the model's convergence order is 1.0 in space.

For comparison with the existing FDAE algorithm, we apply the following implicit difference scheme at the discrete point $\left(x_{n}, t^{k+1}\right)[44]$ :

$$
\begin{aligned}
\frac{u_{n}^{k+1}-u_{n}^{k}}{\Delta t}= & A \frac{(\Delta t)^{-1+\gamma}}{\Gamma(-1+\gamma)} \sum_{i=0}^{k+1} \frac{\Gamma(i+1+\gamma)}{\Gamma(i+1)} \\
& \cdot\left(\frac{u_{n+1}^{k+1-i}-2 u_{n}^{k+1-i}+u_{n-1}^{k+1-i}}{(\Delta x)^{2}}-\frac{u_{n+1}^{k+1-i}-u_{n-1}^{k+1-i}}{2 \Delta x}\right)+q_{n}^{k+1} .
\end{aligned}
$$

The comparison of the infinity norm of absolute errors $\left\|E_{a}\right\|_{\infty}$ for $x=0.5$ between our model and the implicit difference scheme is shown in Table 1, where the infinity norm of absolute errors $\left\|E_{a}\right\|_{\infty}$ is defined as follows:

$$
\left\|E_{a}\right\|_{\infty}(\Delta x, \Delta t)=\max _{1 \leq k \leq T}\left|u^{E}\left(x_{n}, t^{k}\right)-u^{N}\left(x_{n}, t^{k}\right)\right| \text {. }
$$

Here, in equation (29), $T$ is the total discrete time; $u^{E}\left(x_{n}, t^{k}\right)$ represents the exact solution at point $\left(x_{n}, t^{k}\right)$; $u^{N}\left(x_{n}, t^{k}\right)$ represents the LB or difference result at discrete point $\left(x_{n}, t^{k}\right)$. Table 1 illustrates that the infinity norm of the absolute errors of the LB model proposed in this paper is larger than that of the implicit difference scheme in equation (28).

We also compute the convergence rate of the LB model and the implicit difference scheme for $x=0.5$ and present the results in Table 2. Here, convergence rate $R(\Delta x, \Delta t)$ is defined as

$$
R(\Delta x, \Delta t)=\frac{\left\|E_{a}\right\|_{\infty}(2 \Delta x, 2 \Delta t)}{\left\|E_{a}\right\|_{\infty}(\Delta x, \Delta t)}
$$

The convergence rate can be used to measure the speed with which the numerical solution converges to the exact solution as the discrete points increase. From Table 2, we can observe that the convergence rates of the LB model and the implicit difference scheme are close to 2.0, which means that if the discrete points doubled, the infinity norm of the absolute errors will be nearly halved.

In addition, we introduce another coefficient $\rho=\left\|E_{a}\right\|_{\infty}((\Delta x, \Delta t) /(\Delta x))$. Coefficient $\rho$ represents the scaling factor of the infinity norm of the absolute errors $\left\|E_{a}\right\|_{\infty}(\Delta x, \Delta t)$ and space step $\Delta x$. In Table 3 , the parameter $\rho$ for the LB model and the implicit difference scheme for $x=0.5$ is given. From the table, it can be clearly seen that the coefficient $\rho$ for the LB model is within the range of $(1.27,1.35)$, and for the implicit difference scheme, the coefficient $\rho$ is within the range of $(0.117,0.124)$ in this example. With the adjustment of the time and space step, the coefficient $\rho$ for the LB model and the implicit difference scheme does not obviously change, which indicates that both the LB models we presented and the implicit difference scheme have a first-order accuracy of the truncation error.

Finally, we list the computation time cost by these two numerical methods in Table 4 . The LB model costs less CPU time because it is an explicit algorithm, which eliminates the trouble of solving linear algebraic equations. It can be foreseen that when the calculation scale increases, the characteristic of the LB model's low time cost will be more obvious. In particular, the LB model has the advantage of algorithmic parallelism, which is suitable for the large-scale calculation of FDAEs in practical engineering problems.

From the comparison performed above, we can see that the infinity norm of absolute errors $\left\|E_{a}\right\|_{\infty}$ for the LB model is larger than that of the implicit difference scheme. The LB model significantly reduces the CPU time cost. In addition, these two numerical methods have the same convergence rate and convergence order. The LB model, as an explicit algorithm, has several advantages over the finite difference scheme (outstanding explicit algorithmic stability, high algorithmic parallelism, simple handling of complex boundaries, etc.). The LB model presented in this paper is an efficient and selectable method for the FDAE.

Example 2. In this example, the following FDAE is simulated:

$$
\frac{\partial u(x, t)}{\partial t}=\left(A \frac{\partial^{1-\gamma}}{\partial t^{1-\gamma}}+B \frac{\partial^{1-\nu}}{\partial t^{1-\gamma}}\right) \frac{\partial^{2}}{\partial x^{2}} u(x, t)+q(x, t) .
$$




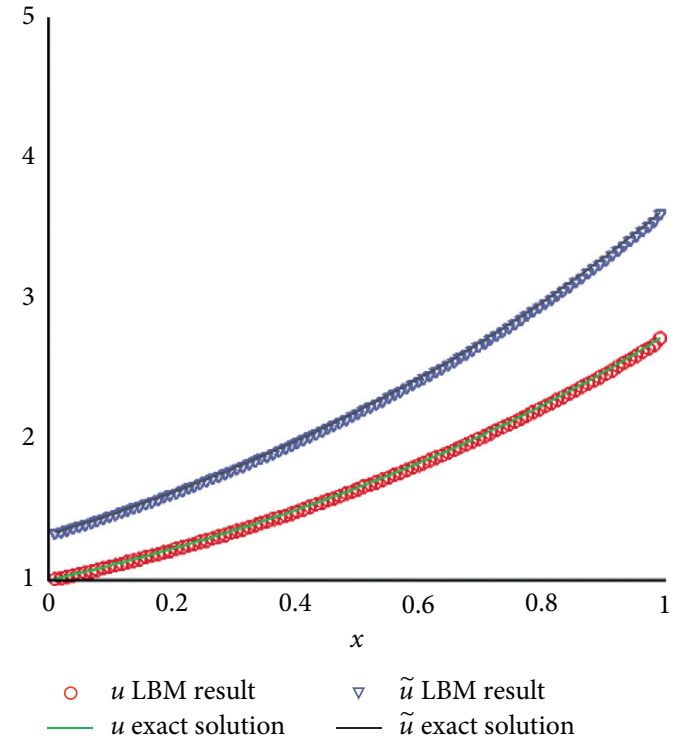

(a)

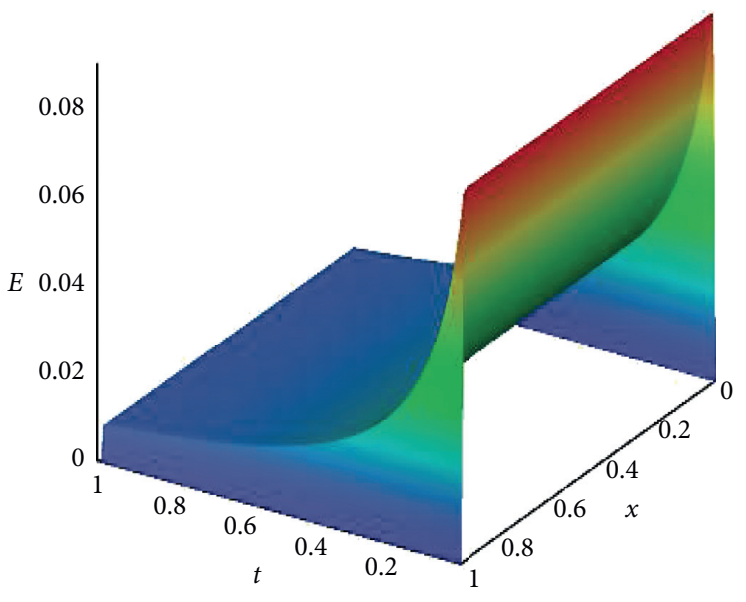

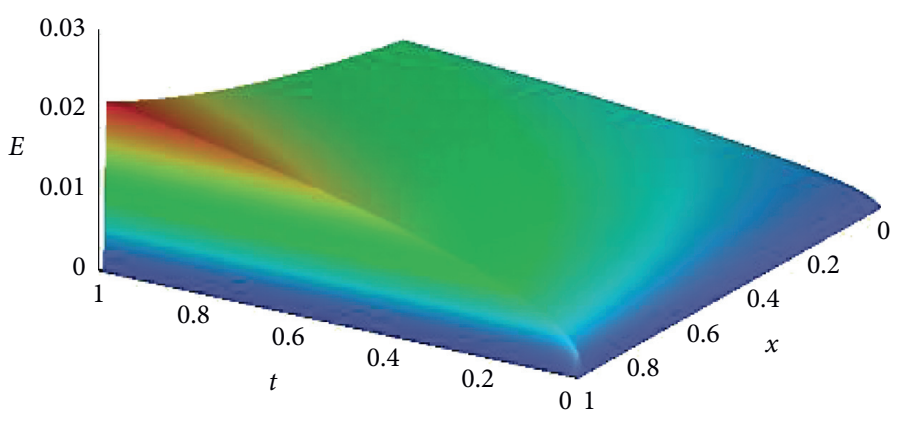

(b)

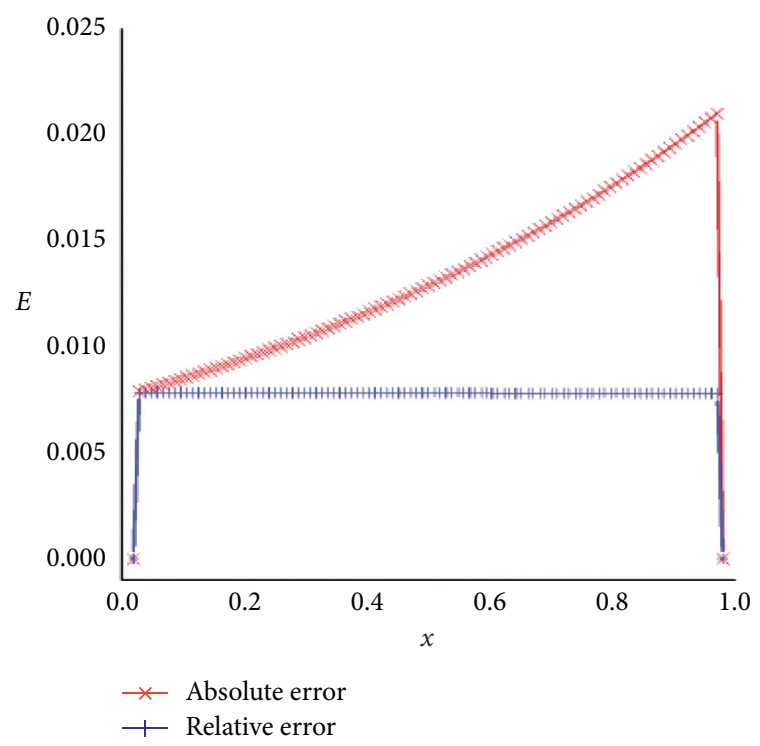

(c)

(d)

Figure 1: Continued. 


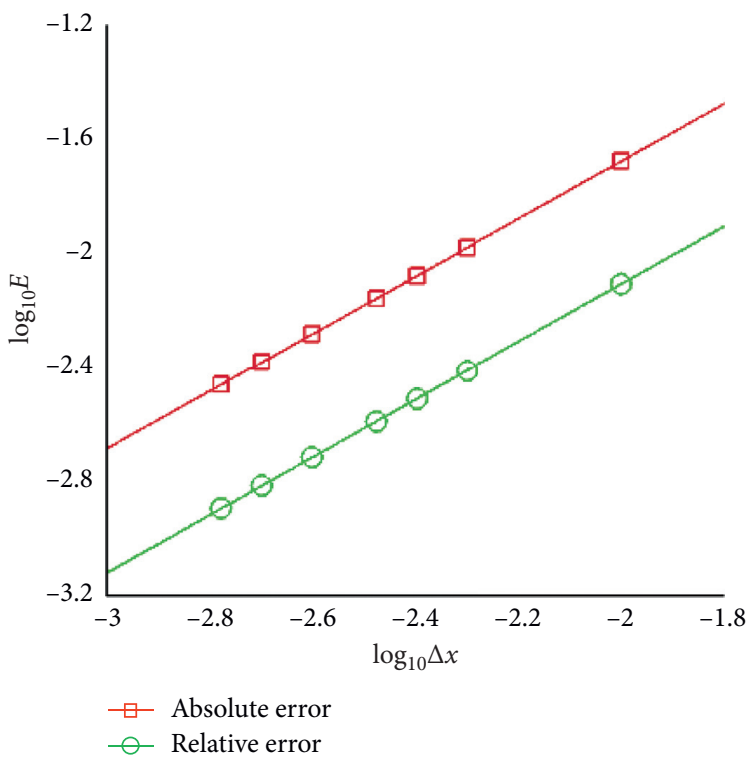

(e)

Figure 1: (a) Comparisons of the exact solutions and LB numerical solutions for $t=1.0$ for Example 1. (b) Surface plot of the absolute errors. (c) Surface plot of the relative errors. (d) Curves of the absolute errors and relative errors for $t=1.0$. (e) Relationship between the errors and lattice size $\Delta x$ for $t=1.0$. The parameters for (a)-(d) are as follows: $\gamma=0.5, M=100, c=1.0, \Delta x=0.01, \Delta t=0.01$, and $\tau=0.908$. The parameters for (e) are as follows: $\gamma=0.5, c=1.0$, and $\tau=0.908$.

TABLE 1: Comparison of the infinity norm of absolute errors $\left\|E_{a}\right\|_{\infty}$ for $x=0.5$ between the LB model and the implicit difference scheme in Example 1. The parameters for the LB model are as follows: $\gamma=0.5, c=1.0$, and $\tau=0.908$.

\begin{tabular}{lcc}
\hline & Our model & Implicit difference scheme equation (28) [44] \\
\hline$\Delta x=\Delta t=0.05$ & $6.731748 \times 10^{-2}$ & $5.892235 \times 10^{-3}$ \\
$\Delta x=\Delta t=0.025$ & $3.288136 \times 10^{-2}$ & $3.013750 \times 10^{-3}$ \\
$\Delta x=\Delta t=0.0125$ & $1.615917 \times 10^{-2}$ & $1.529798 \times 10^{-3}$ \\
$\Delta x=\Delta t=0.00625$ & $7.978385 \times 10^{-3}$ & $7.727516 \times 10^{-4}$ \\
\hline
\end{tabular}

TABLE 2: The convergence rate $R(\Delta x, \Delta t)$ for the LB model presented in this paper and the implicit difference scheme for $x=0.5$ in Example 1. The parameters for the LB model are the same as those in Table 1.

\begin{tabular}{lcc}
\hline & Our model & Implicit difference scheme equation (28) \\
\hline$\Delta x=\Delta t=0.025$ & 2.047284 & 1.955117 \\
$\Delta x=\Delta t=0.0125$ & 2.034842 & 1.970031 \\
$\Delta x=\Delta t=0.00625$ & 2.025369 & 1.979676 \\
\hline
\end{tabular}

TABLE 3: The parameter $\rho$ for the LB model presented in this paper and the implicit difference scheme for $x=0.5$ in Example 1 . The parameters for the LB model are the same as those in Table 1.

\begin{tabular}{lcc}
\hline & Our model & Implicit difference scheme equation (28) \\
\hline$\Delta x=\Delta t=0.05$ & 1.346350 & 0.1178447 \\
$\Delta x=\Delta t=0.025$ & 1.315254 & 0.1205500 \\
$\Delta x=\Delta t=0.0125$ & 1.292734 & 0.1223838 \\
$\Delta x=\Delta t=0.00625$ & 1.276542 & 0.1236403 \\
\hline
\end{tabular}

TABLE 4: The comparison of the time cost of the LB model presented in this paper and the implicit difference in Example 1. The parameters for the LB model are the same as those in Table 1.

\begin{tabular}{ccc}
\hline & Our model $(\mathrm{s})$ & Implicit difference scheme equation $(28)$ \\
\hline$\Delta x=\Delta t=0.0025$ & $2.5 \mathrm{~s}$ & $5.3 \mathrm{~s}$ \\
\hline
\end{tabular}


The parameters are $\gamma=0.5, \nu=0.6$, and $A=B=0.001$. Variables $x, t \in[0,1]$. The source term $q(x, t)$ is given by

$$
q(x, t)=2 e^{x}\left(t-A \frac{t^{1+\gamma}}{\Gamma(2+\gamma)}-B \frac{t^{1+\nu}}{\Gamma(2+\nu)}\right) .
$$

The initial condition is given by

$$
u(x, 0)=0 .
$$

The Dirichlet boundary conditions are given by the following equations:

$$
\begin{gathered}
u(0, t)=t^{2}, \\
u(1, t)=e t^{2}
\end{gathered}
$$

The exact solution to this problem is given as follows:

$$
u(x, t)=e^{x} t^{2} .
$$

From equations (5a)-(5b), we can obtain the initial and boundary conditions of variables $\widetilde{u}(x, t)$ and $\bar{u}(x, t)$ :

$$
\begin{gathered}
\tilde{u}(x, 0)=0, \\
\tilde{u}(0, t)=\frac{\Delta t^{-1+\gamma}}{\Gamma(-1+\gamma)} \sum_{i=0}^{[t / \Delta t]} \frac{\Gamma(i-1+\gamma)}{\Gamma(i+1)}(t-i \Delta t)^{2}, \\
\widetilde{u}(1, t)=\frac{\Delta t^{-1+\gamma}}{\Gamma(-1+\gamma)} \sum_{i=0}^{[t / \Delta t]} \frac{\Gamma(i-1+\gamma)}{\Gamma(i+1)}(t-i \Delta t)^{2} e . \\
\bar{u}(x, 0)=0, \\
\bar{u}(0, t)=\frac{\Delta t^{-1+v}}{\Gamma(-1+v)} \sum_{i=0}^{[t / \Delta t]} \frac{\Gamma(i-1+\nu)}{\Gamma(i+1)}(t-i \Delta t)^{2}, \\
\bar{u}(1, t)=\frac{\Delta t^{-1+v}}{\Gamma(-1+v)} \sum_{i=0}^{[t / \Delta t]} \frac{\Gamma(i-1+\nu)}{\Gamma(i+1)}(t-i \Delta t)^{2} e .
\end{gathered}
$$

By employing equation (3a) and (3b), we can derive the exact solutions of $\widetilde{u}(x, t)$ and $\bar{u}(x, t)$ :

$$
\begin{aligned}
& \tilde{u}(x, t)=\frac{2 e^{x} t^{1+\gamma}}{\Gamma(2+\gamma)}, \\
& \bar{u}(x, t)=\frac{2 e^{x} t^{1+v}}{\Gamma(2+\nu)} .
\end{aligned}
$$

The numerical results are shown in Figure 2. In Figure 2(a), comparisons of the exact solutions and the LB numerical results for $u(x, t), \widetilde{u}(x, t)$, and $\bar{u}(x, t)$ for $t=1.0$ are presented. The parameters are $\gamma=0.5, v=0.6$, total lattice number $M=100, \Delta x=0.01, \Delta t=0.01, c=1.0$, and $\tau=0.908$. The graph illustrates that the LB numerical results are consistent with the exact solutions.
Figures 2(b) and 2(c) show the surface plots of absolute errors $E_{a}$ and relative errors $E_{r}$, respectively. These two graphs show that the absolute errors are less than $3.0 \times 10^{-2}$, whereas the relative errors are consistently lower than $8.0 \times$ $10^{-2}$ from $t=0.1$ to $t=1.0$.

The relationship between the errors and spatial positions $x$ for $t=1.0$ is plotted in Figure 2(d). The parameters are the same as those shown in Figure 2(a). From Figure 2(d), we can observe that the absolute errors $E_{a}$ are less than 0.03 , while the relative errors $E_{r}$ are close to 0.01 for $t=1.0$.

Example 3. Finally, the following form of FDAE is considered:

$$
\frac{\partial u(x, t)}{\partial t}=A \frac{\partial^{1-\gamma}}{\partial t^{1-\gamma}} \frac{\partial^{2}}{\partial x^{2}} u(x, t)-A \frac{\partial^{1-\gamma}}{\partial t^{1-\gamma}} \frac{\partial}{\partial x} u(x, t)+q(x, t) .
$$

The parameters are $A=0.001$ and $0<\gamma<1$. The variables $x, t \in[0,1]$. The source term is $q(x, t)$ (see equation (39) for their relationship):

$$
q(x, t)=x^{2}(1-x) t^{\gamma}(1+\gamma)-A \frac{\Gamma(2+\gamma)}{\Gamma(1+\gamma)} t^{2 \gamma}\left(3 x^{2}-8 x+2\right) .
$$

The initial condition of the problem is given by

$$
u(x, 0)=0 .
$$

The Dirichlet boundary conditions are given by

$$
\begin{aligned}
& u(0, t)=0, \\
& u(1, t)=0 .
\end{aligned}
$$

The exact solution to the problem is

$$
u(x, t)=x^{2}(1-x) t^{\gamma+1} .
$$

By applying equations (5a) and (3a) as mentioned in Example 1 and Example 2, we can obtain the initial and boundary conditions of $\widetilde{u}(x, t)$ as well as the exact solutions of $\widetilde{u}(x, t)$ :

$$
\begin{aligned}
& \widetilde{u}(x, 0)=0, \\
& \widetilde{u}(0, t)=0, \\
& \widetilde{u}(1, t)=0, \\
& \widetilde{u}(x, t)=\frac{\Gamma(\gamma+2)}{\Gamma(2 \gamma+1)} x^{2}(1-x) t^{\gamma+2} .
\end{aligned}
$$

In Figure 3(a), the LB numerical results and exact solutions for $t=1.0$ are presented. The parameters are selected as $\gamma=0.4$, total lattice number $M=100, \Delta x=0.01$, $\Delta t=0.01, c=1.0$, and $\tau=0.908$. We can see that the 


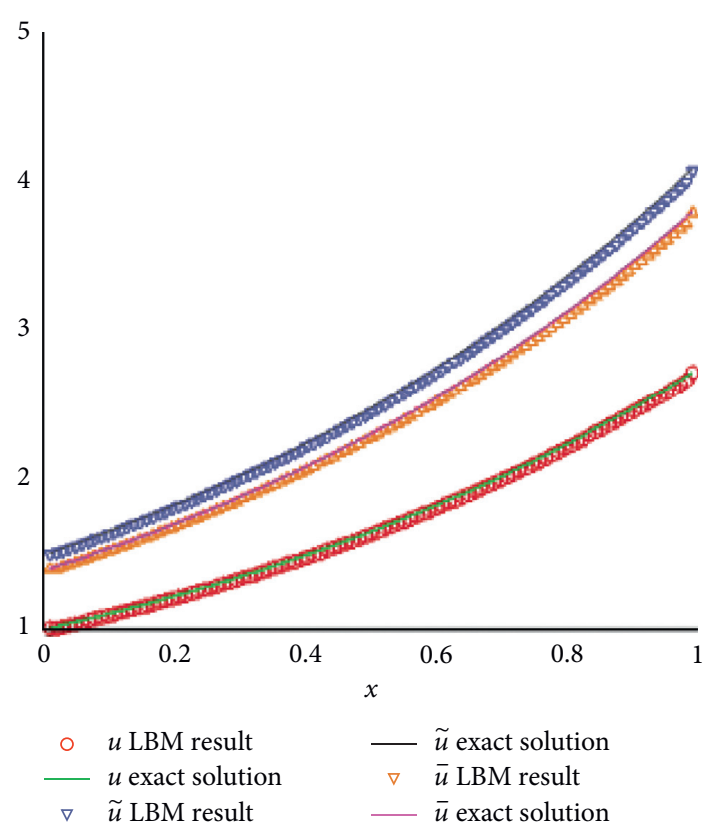

(a)

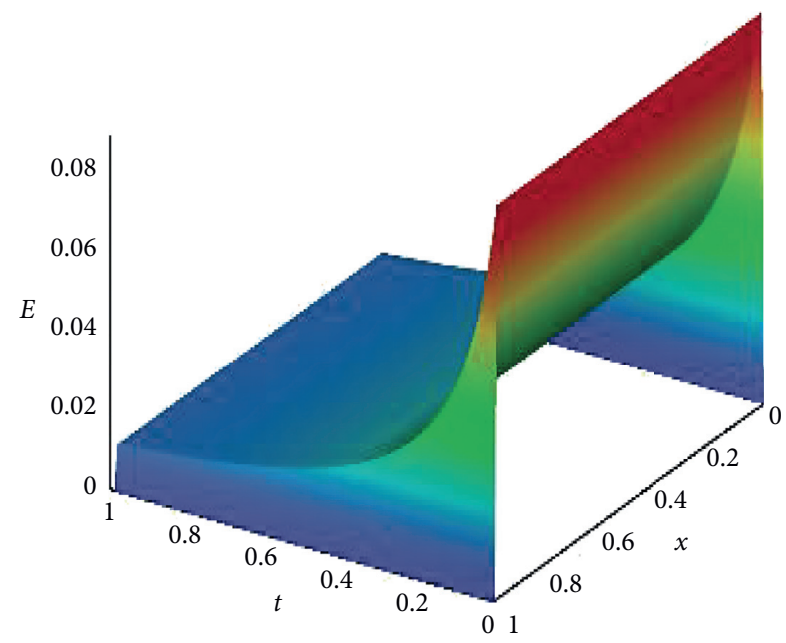

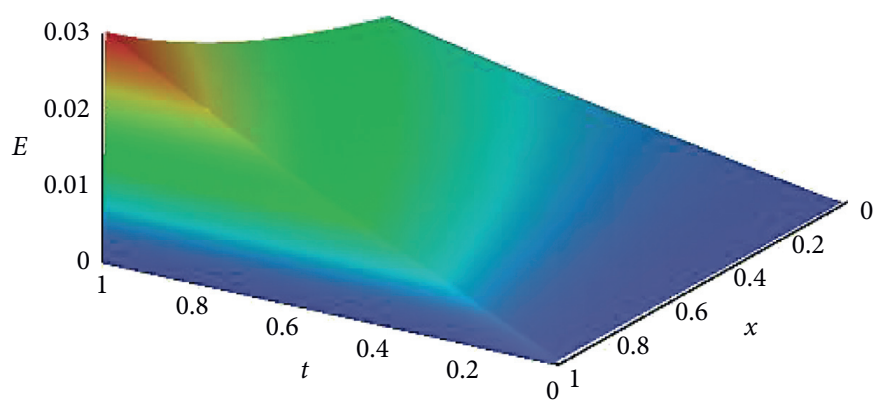

(b)

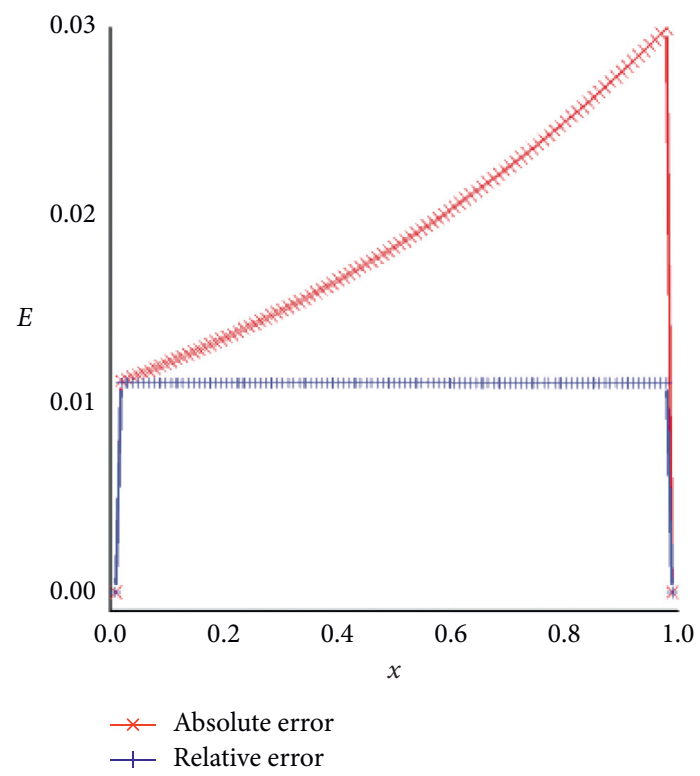

(d)

Figure 2: (a) Comparisons of the exact and LB numerical results for $u(x, t), \tilde{u}(x, t)$, and $\bar{u}(x, t)$ for $t=1.0$. (b) The surface plot of the absolute errors. (c) The surface plot of the relative errors. (d) Relationship between the errors and spatial positions $x$ for $t=1.0$. The parameters are $\gamma=0.5, \nu=0.6$, total lattice number $M=100, \Delta x=0.01, \Delta t=0.01, c=1.0$, and $\tau=0.908$.

numerical and exact solutions are in good agreement. The surface plots of the absolute errors and relative errors in this example are plotted in Figures 3(b) and 3(c) with time $t \in[0.2,1.0]$ and $x \in[0.2,0.8]$. In Figure $3(\mathrm{~d})$, the absolute errors and relative errors are presented for $t=1.0$. The graphs from Figures 3(b) to 3(d) illustrate that the absolute errors are less than $1.0 \times 10^{-3}$, whereas the relative errors are consistently less than $5.0 \times 10^{-2}$. When $t=1.0, E_{a}<0.001$ and $E_{r}<0.02$. The numerical results presented above imply that our model is suitable for simulating FDAEs. 


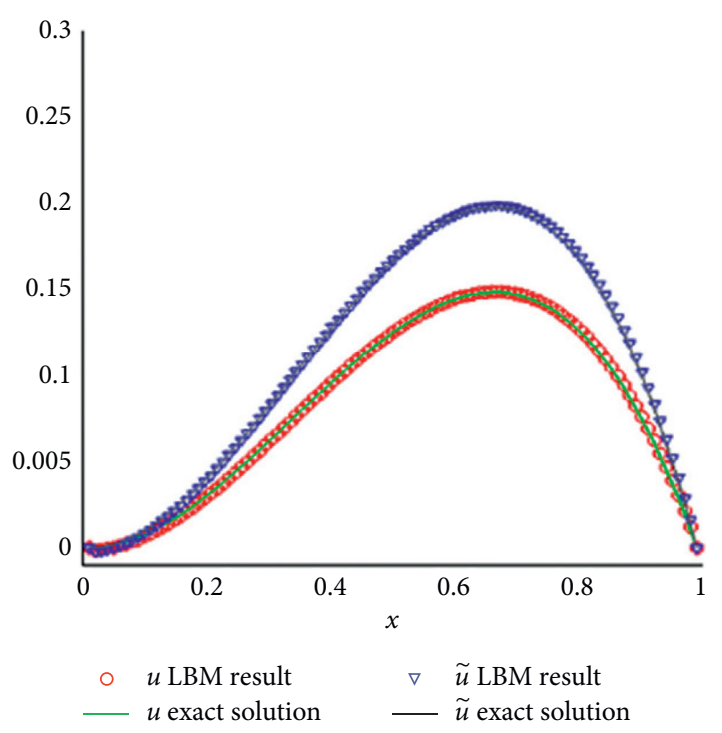

(a)

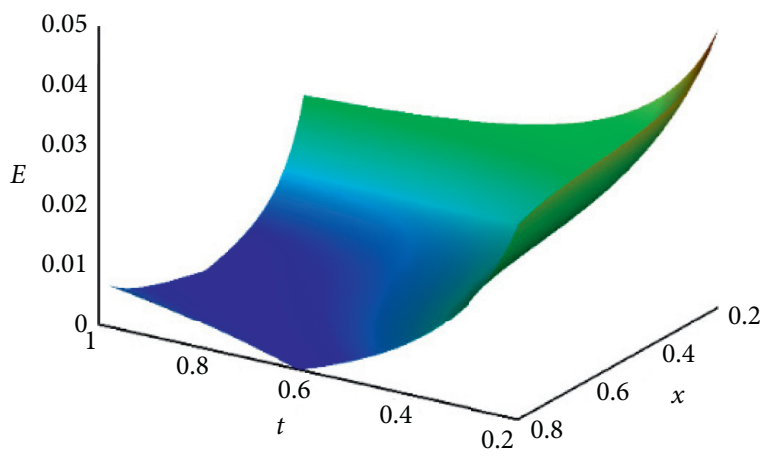

(c)

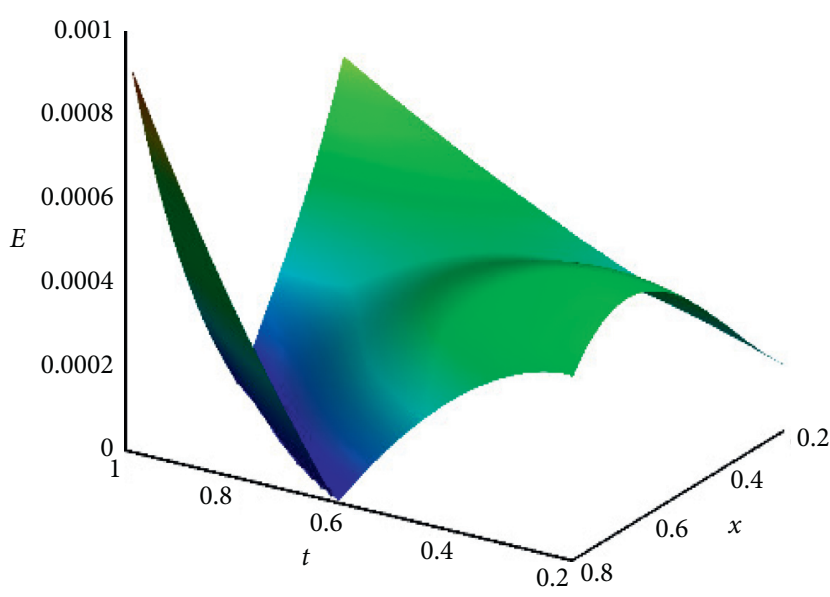

(b)

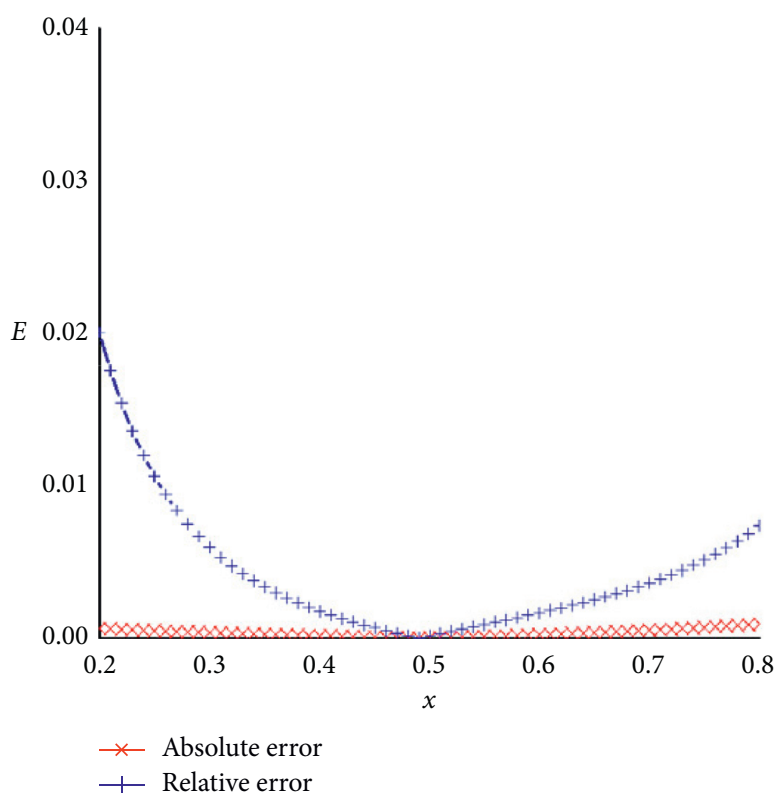

(d)

Figure 3: (a) Comparisons of the LB numerical results and exact solutions for $t=1.0$. (b) The surface plot of absolute errors. (c) The surface plot of relative errors. (d) The absolute errors and relative errors for $t=1.0$. Parameters are $\gamma=0.4$, total lattice number $M=100, \Delta x=0.01$, $\Delta t=0.01, c=1.0$, and $\tau=0.908$.

\section{Conclusions}

In this paper, an LB model is proposed to investigate the FDAE. The Riemann-Liouville definition and the Grünwald-Letnikov definition are applied to the timefractional derivatives. Then, a simple LB model is presented to solve the equation. By introducing the Chapman-Enskog analysis, the FDAE with second-order accuracy of the truncation error is recovered. The model is efficient and promising for future practical applications, not only because the introduction of the Grünwald-Letnikov definition simplifies the calculations of time-fractional derivatives but also because the model retains advantages of the LB method over traditional numerical methods. Three numerical examples are carried out to test the validity of our model. The close agreement between the numerical results and the exact solutions implies that our model is suitable for simulating FDAE. In addition, a comparative study is also conducted. The results demonstrate that the proposed model and the implicit finite difference scheme have the same convergence rate and convergence order. The LB model has a larger absolute error but costs less CPU time than the implicit finite difference scheme.

However, some aspects of the model are less successful and still need further research. The accuracy of the model is unsatisfactory. We think there are two possible reasons for 
the low spatial accuracy: (1) the treatment of the fractional derivative terms and (2) the introduction of additional distribution functions $\Omega_{\alpha}^{(n)}$. We are still looking for a better way to improve the accuracy of the Grünwald-Letnikov definition. In addition, constructing more effective and accurate additional distribution functions for the source terms in the FDAEs is an ongoing challenge and still needs further research.

\section{Appendix}

In this section, the detailed recovery process of the FDAE is provided. First, we take equation (13a) and sum over $\alpha$ :

$$
\frac{\partial u}{\partial t_{0}}+\frac{\partial m}{\partial x}=\sum_{\alpha} \Omega_{\alpha}^{(1)}
$$

Now, based on equation (A.1), we take $(13 a)+(13 b) \times \mathcal{E}$ and sum over $\alpha$ :

$$
\begin{aligned}
\frac{\partial u}{\partial t}+ & \frac{\partial m^{(0)}}{\partial x}+\varepsilon C_{2} \frac{\partial^{2} \pi^{(0)}}{\partial x^{2}} \\
= & \sum_{\alpha}\left(\Omega_{\alpha}^{(1)}+\varepsilon \Omega_{\alpha}^{(2)}-\varepsilon \tau \Delta \Omega_{\alpha}^{(1)}-\varepsilon C_{2} \frac{\partial \Omega_{\alpha}^{(1)}}{\partial t}\right) \\
& -\varepsilon C_{2} \frac{\partial^{2} m^{(0)}}{\partial t \partial x}+O\left(\varepsilon^{2}\right) .
\end{aligned}
$$

Substituting equations (17a) and (17b) into equation (A.2), we have

$$
\begin{aligned}
\frac{\partial u}{\partial t}+F \frac{\partial \widetilde{u}}{\partial x}= & \frac{\partial^{2}(A \widetilde{u}+B \bar{u})}{\partial x^{2}} \\
& +\sum_{\alpha}\left(\Omega_{\alpha}^{(1)}+\varepsilon \Omega_{\alpha}^{(2)}-\varepsilon \tau \Delta \Omega_{\alpha}^{(1)}-\varepsilon C_{2} \frac{\partial \Omega_{\alpha}^{(1)}}{\partial t}\right) \\
& -\varepsilon C_{2} F \frac{\partial^{2} \widetilde{u}}{\partial t \partial x}+O\left(\varepsilon^{2}\right) .
\end{aligned}
$$

If we choose the source term

$$
\begin{aligned}
q(x, t)= & \sum_{\alpha}\left(\Omega_{\alpha}^{(1)}+\varepsilon \Omega_{\alpha}^{(2)}-\varepsilon \tau \Delta \Omega_{\alpha}^{(1)}-\varepsilon C_{2} \frac{\partial \Omega_{\alpha}^{(1)}}{\partial t}\right) \\
& -\varepsilon C_{2} F \frac{\partial^{2} \widetilde{u}}{\partial t \partial x}
\end{aligned}
$$

then equation (A.3) can be written as

$$
\frac{\partial u}{\partial t}+F \frac{\partial \widetilde{u}}{\partial x}=\frac{\partial^{2}}{\partial x^{2}}(A \widetilde{u}+B \bar{u})+q+O\left(\varepsilon^{2}\right) .
$$

This is the FDAE with second-order accuracy of the truncation error.

From equation (A.4), we can see that the additional distribution functions $\Omega_{\alpha}^{(1)}$ and $\Omega_{\alpha}^{(2)}$ are related to the source term $q(x, t)$ and the variable $\widetilde{u}(x, t)$. For the D1Q3 model, it is assumed that $\Omega_{\alpha}^{(1)}=0$. Therefore, $\Omega_{\alpha}^{(2)}$ can be obtained:

$$
\Omega_{\alpha}^{(2)}=\varphi=\frac{1}{3 \varepsilon}\left[q(x, t)+\varepsilon C_{2} F \frac{\partial^{2} \widetilde{u}}{\partial t \partial x}\right] . \quad \alpha=0,1,2 .
$$

In equation (A.6), the term $\varepsilon C_{2} F\left(\partial^{2} \widetilde{u} / \partial t \partial x\right)$ can be calculated by applying the difference scheme:

$$
\varepsilon C_{2} F \frac{\partial^{2} \widetilde{u}}{\partial t \partial x}=\frac{\varepsilon C_{2} F}{2 \Delta x}\left(\frac{\widetilde{u}_{n+1}^{k}-\widetilde{u}_{n+1}^{k-1}}{\Delta t}-\frac{\widetilde{u}_{n-1}^{k}-\widetilde{u}_{n-1}^{k-1}}{\Delta t}\right) .
$$

\section{Data Availability}

The data used to support the findings of this study are available from the corresponding author upon request.

\section{Conflicts of Interest}

The authors declare that they have no conflicts of interest.

\section{Acknowledgments}

The authors would like to dedicate this article to Prof. Guangwu Yan, who unfortunately passed away before this paper was submitted to the journal. The authors will miss him forever! This work was supported by the National Nature Science Foundation of China (grant nos. 11272133 and 11602033).

\section{References}

[1] Z. Liu, Y. Zhu, J. R. Clausen, J. B. Lechman, R. R. Rao, and C. K. Aidun, "Multiscale method based on coupled latticeBoltzmann and Langevin-dynamics for direct simulation of nanoscale particle/polymer suspensions in complex flows," International Journal for Numerical Methods in Fluids, vol. 91, no. 5, pp. 228-246, 2019.

[2] T. Kataoka and T. Hanada, "New lattice Boltzmann model for the compressible Navier-Stokes equations," International Journal for Numerical Methods in Fluids, vol. 91, no. 4, pp. 183-197, 2019.

[3] M. Ashna, M. H. Rahimian, and A. Fakhari, "Extended lattice Boltzmann scheme for droplet combustion," Physical Review E, vol. 95, Article ID 053301, 2017.

[4] A. M. Velasco, J. D. Muñoz, and M. Mendoza, "Lattice Boltzmann model for the simulation of the wave equation in curvilinear coordinates," Journal of Computational Physics, vol. 376, pp. 76-97, 2019.

[5] D. Li, H. Lai, and B. Shi, "Mesoscopic simulation of the $(2+$ 1)-dimensional wave equation with nonlinear damping and source terms using the lattice Boltzmann BGK model," Entropy, vol. 21, no. 4, p. 390, 2019.

[6] W.-Q. Hu and S.-L. Jia, "General propagation lattice Boltzmann model for variable-coefficient non-isospectral $\mathrm{KdV}$ equation," Applied Mathematics Letters, vol. 91, pp. 61-67, 2019.

[7] H. Otomo, B. M. Boghosian, and F. Dubois, "Efficient lattice Boltzmann models for the Kuramoto-Sivashinsky equation," Computers \& Fluids, vol. 172, pp. 683-688, 2018.

[8] Z. Chai, B. Shi, and Z. Guo, "A multiple-relaxation-time lattice Boltzmann model for general nonlinear anisotropic convection-diffusion equations," Journal of Scientific Computing, vol. 69, no. 1, pp. 355-390, 2016.

[9] H. Wang and G. Yan, "Lattice Boltzmann model for the interaction of $(2+1)$-dimensional solitons in generalized 
Gross-Pitaevskii equation," Applied Mathematical Modelling, vol. 40, no. 7-8, pp. 5139-5152, 2016.

[10] H. Wang, "Numerical simulation for the Gross-Pitaevskii equation based on the lattice Boltzmann method," Advances in Space Research, vol. 60, no. 6, pp. 1261-1270, 2017.

[11] S. Succi, "Numerical solution of the Schrödinger equation using discrete kinetic theory," Physical Review E, vol. 53, no. 2, pp. 1969-1975, 1996.

[12] L. H. Zhong, S. D. Feng, P. Dong, and S. T. Gao, "Lattice Boltzmann schemes for the nonlinear Schrödinger equation," Physical Review E, vol. 74, Article ID 036704, 2006.

[13] J. Zhang and G. Yan, "A lattice Boltzmann model for the nonlinear Schrödinger equation," Journal of Physics A: Mathematical and Theoretical, vol. 40, no. 33, pp. 1039310405, 2007.

[14] J. Zhang and G. Yan, "Lattice Boltzmann model for complex Ginzburg-Landau equation in curvilinear coordinates," Computers \& Mathematics with Applications, vol. 70, no. 12, pp. 2904-2919, 2015.

[15] J. Zhang, G. Yan, and M. Wang, "Lattice Boltzmann simulations for the vortex tori pattern in the three-dimensional cubic-quintic complex Ginzburg-Landau equation," Journal of Computational Physics, vol. 306, pp. 311-319, 2016.

[16] R. R. Nigmatullin, "The realization of the generalized transfer equation in a medium with fractal geometry," Physica Status Solidi (B), vol. 133, no. 1, pp. 425-430, 1986.

[17] R. Metzler and J. Klafter, "The random walk's guide to anomalous diffusion: a fractional dynamics approach," Physics Reports, vol. 339, no. 1, pp. 1-77, 2000.

[18] I. M. Sokolova and J. Klafter, "From diffusion to anomalous diffusion: a century after Einstein's Brownian motion," Chaos, vol. 15, Article ID 026103, 2005.

[19] S. B. Yuste, L. Acedo, and K. Lindenberg, "Reaction front in an $\mathrm{A}+\mathrm{B} \longrightarrow \mathrm{C}$ reaction-subdiffusion process," Physical Review E, vol. 69, Article ID 036126, 2004.

[20] B. Bodo, A. Mvogo, and S. Morfu, "Fractional dynamical behavior of electrical activity in a model of pancreatic $\beta$-cells," Chaos, Solitons \& Fractals, vol. 102, pp. 426-432, 2017.

[21] C. M. A. Pinto and J. A. T. Machado, "Fractional dynamics of computer virus propagation," Mathematical Problems in Engineering, vol. 2014, Article ID 476502, 7 pages, 2014.

[22] P. Muthukumar and P. Balasubramaniam, "Feedback synchronization of the fractional order reverse butterfly-shaped chaotic system and its application to digital cryptography," Nonlinear Dynamics, vol. 74, no. 4, pp. 1169-1181, 2013.

[23] A. Kiani-B, K. Fallahi, N. Pariz, and H. Leung, "A chaotic secure communication scheme using fractional chaotic systems based on an extended fractional Kalman filter," Communications in Nonlinear Science and Numerical Simulation, vol. 14, no. 3, pp. 863-879, 2009.

[24] A. Jajarmi, M. Hajipour, and D. Baleanu, "New aspects of the adaptive synchronization and hyperchaos suppression of a financial model," Chaos, Solitons \& Fractals, vol. 99, pp. 285-296, 2017.

[25] M. Bishehniasar, S. Salahshour, A. Ahmadian, F. Ismail, and D. Baleanu, "An accurate approximate-analytical technique for solving time-fractional partial differential equations," Complexity, vol. 2017, Article ID 8718209, 12 pages, 2017.

[26] C. Tadjeran and M. M. Meerschaert, "A second-order accurate numerical method for the two-dimensional fractional diffusion equation," Journal of Computational Physics, vol. 220, no. 2, pp. 813-823, 2007.

[27] M. Hajipour, A. Jajarmi, and D. Baleanu, "An efficient nonstandard finite difference scheme for a class of fractional chaotic systems," Journal of Computational and Nonlinear Dynamics, vol. 13, Article ID 021013, 2018.

[28] A. Ahmadian, F. Ismail, S. Salahshour, D. Baleanu, and F. Ghaemi, "Uncertain viscoelastic models with fractional order: a new spectral tau method to study the numerical simulations of the solution," Communications in Nonlinear Science and Numerical Simulation, vol. 53, pp. 44-64, 2017.

[29] A. Ahmadian, S. Salahshour, M. Ali-Akbari, F. Ismail, and D. Baleanu, "A novel approach to approximate fractional derivative with uncertain conditions," Chaos, Solitons \& Fractals, vol. 104, pp. 68-76, 2017.

[30] G. Acosta and J. P. Borthagaray, "A fractional Laplace equation: regularity of solutions and finite element approximations," SIAM Journal on Numerical Analysis, vol. 55, no. 2, pp. 472-495, 2017.

[31] L. B. Feng, P. Zhuang, F. Liu, and I. Turner, "Stability and convergence of a new finite volume method for a two-sided space-fractional diffusion equation," Applied Mathematics and Computation, vol. 257, pp. 52-65, 2015.

[32] X. Sun, C. Li, and F. Zhao, "Local discontinuous Galerkin methods for the time tempered fractional diffusion equation," Applied Mathematics and Computation, vol. 365, Article ID 124725, 2020.

[33] Q. Liu, Y. T. Gu, P. Zhuang, F. Liu, and Y. F. Nie, "An implicit RBF meshless approach for time fractional diffusion equations," Computational Mechanics, vol. 48, no. 1, pp. 1-12, 2011.

[34] Y. Xia, J. Wu, and Y. Zhang, "Lattice-Boltzmann simulation of two-dimensional super-diffusion," Engineering Applications of Computational Fluid Mechanics, vol. 6, no. 4, pp. 581-594, 2012.

[35] J. Y. Zhang and G. W. Yan, "Lattice Boltzmann method for the fractional sub-diffusion equation," International Journal for Numerical Methods in Fluids, vol. 80, pp. 490-507, 2015.

[36] J. G. Zhou, P. M. Haygarth, P. J. A. Withers, C. J. A. Macleod, P. D. Falloon et al., "Lattice Boltzmann method for the fractional advection-diffusion equation," Physical Review E, vol. 93, Article ID 043310, 2016.

[37] H.-P. Dai, Z.-S. Zheng, and W. Tan, "Lattice Boltzmann model for the Riesz space fractional reaction-diffusion," Thermal Science, vol. 22, no. 4, pp. 1831-1843, 2018.

[38] F. Wang, X. Zhang, X. Shen, and J. Sun, “A Lattice Boltzmann model for 2D fractional advection-dispersion equation: theory and application," Journal of Hydrology, vol. 564, pp. 246-255, 2018.

[39] A. Cartalade, A. Younsi, and M.-C. Néel, "Multiple-Relaxation-Time Lattice Boltzmann scheme for fractional advectiondiffusion equation," Computer Physics Communications, vol. 234, pp. 40-54, 2019.

[40] R. Du, D. Sun, B. Shi, and Z. Chai, "Lattice Boltzmann model for time sub-diffusion equation in Caputo sense," Applied Mathematics and Computation, vol. 358, pp. 80-90, 2019.

[41] R. Du and Z. Liu, "A lattice Boltzmann model for the fractional advection-diffusion equation coupled with incompressible Navier-Stokes equation," Applied Mathematics Letters, vol. 101, Article ID 106074, 2020.

[42] S. B. Yuste and K. Lindenberg, "Subdiffusion-limited A+A reactions," Physical Review Letters, vol. 87, p. 118301, 2001.

[43] S. Chapman and T. G. Cowling, The Mathematical Theory of Non-uniform Gases, Cambridge University, Cambridge, UK, 1970.

[44] Z. Q. Ding, “An implicit finite difference method for a class of fractional dispersion advection equations," Journal of Hunan University of Arts and Science (Natural Science Edition), vol. 21, pp. 3-5, 2009, in Chinese. 